\title{
As três emergências que nossa sociedade enfrenta: saúde, biodiversidade e mudanças climáticas
}

PAULO ARTAXO ${ }^{I}$

\section{Introdução}

$N$

OSSA SOCIEDADE está simultaneamente convivendo com três emergências importantes: 1) a crise na saúde; 2) a crise de perda de biodiversidade; e 3) a crise climática. Salienta-se que essas crises têm ligações profundas entre si, e diferenças importantes, mas todas provocam impactos sociais e econômicos fortes e afetam nosso planeta globalmente.

Entre os pontos comuns mais relevantes cita-se um modelo de desenvolvimento econômico que é orientado pelo maior lucro no menor espaço de tempo, não importando as consequências futuras. Outro ponto comum importante é que, nas três crises, em geral, governos vão contra as recomendações da ciência. A ciência coloca há mais de duas décadas que uma pandemia como a da Covid-19 poderia chegar e atingir nossa sociedade com pesados prejuízos socioeconômicos. A ciência também vem alertando para o risco das mudanças climáticas, desde muito antes da Conferência Rio-92, principalmente sobre os riscos que a perda da biodiversidade provoca em muitas áreas da vida no planeta. Existem milhares de vírus desconhecidos da ciência que ainda estão em equilíbrio na flora e fauna. Todavia, com a continuidade da perda das florestas tropicais por meio do desmatamento, é só questão de tempo para que um novo vírus migre da Amazônia para outras regiões, com prejuízos enormes, como os causados pelo Sars-CoV-2.

Entre as diferenças importantes nas três crises está a questão temporal: enquanto a crise na saúde associada ao Sars-CoV-2 pode durar cerca de dois anos, o impacto das mudanças climáticas pode durar séculos, a perda de biodiversidade é para sempre. Não há possível lockdown como aplicado na pandemia da Covid-19, nas crises climáticas e de biodiversidade.

Importante salientar que as mudanças climáticas têm fortes ligações com a perda da biodiversidade que observamos em praticamente todos os ecossistemas terrestres, aquáticos e no ambiente marinho (IPBES 2019). A resiliência dos ecossistemas e sua capacidade de reagir a mudanças dependem, em grande par- 
te, de sua biodiversidade. As alterações observadas na taxa de precipitação e sua sazonalidade, e no aumento de temperatura, estão afetando o funcionamento dos ecossistemas. As mudanças climáticas afetam os padrões ecossistêmicos da fotossíntese e da produtividade, podendo modificar os ciclos hidrológicos e a dinâmica do carbono em ecossistemas terrestres e marinhos.

O funcionamento de nossos ecossistemas está sendo fortemente afetado pelas mudanças climáticas, não somente aquelas em nível global, mas, também, regional e até mesmo local. Nas florestas tropicais, o aumento da produção de biomassa, mortalidade das árvores, alterações na distribuição e abundância de espécies e incêndios estão entre as mudanças relacionadas à elevação de $\mathrm{CO}_{2}$ atmosférico. Considerando os efeitos sinérgicos da mudança do uso do solo, do desmatamento e o elevado grau de fragmentação e degradação da maior parte dos biomas brasileiros, a vulnerabilidade da nossa biota e ecossistemas aumenta, ameaçando a biodiversidade e os serviços ecossistêmicos associados.

Tal é a dimensão das alterações determinadas pela ação humana no planeta, que a comunidade científica considera esta como uma nova era geológica, o Antropoceno, que está alterando profundamente nossa relação com a natureza. É fundamental, portanto, desenvolvermos uma ciência que contribua para alcançarmos uma sociedade sustentável ao longo das próximas décadas, conciliando preservação do meio ambiente com desenvolvimento econômico e justiça social.

O Brasil é signatário do Acordo de Paris e dos Objetivos do Desenvolvimento Sustentável (ODS) (Agenda 2030, ONU Brasil) e sua comunidade científica tem contribuindo fortemente com avanços científicos capazes de pautar as esferas das políticas públicas e estratégias de desenvolvimento socioeconômico, e também de orientar os tomadores de decisão quanto ao desenvolvimento sustentável do país. O Brasil precisa enfrentar os desafios impostos pela alteração climática e ambiental, que passam pelo estabelecimento de métricas e referências, de forma a contribuir na formulação de estratégias regionais e nacionais de detecção/atribuição, mitigação e adaptação aos seus efeitos. O atendimento dos ODS e das metas do Acordo de Paris é um passo importante no caminho da sustentabilidade, mas não suficiente. Temos um longo caminho para a construção de uma nova sociedade que seja minimamente sustentável.

O crescimento da população humana mundial, que poderá alcançar entre 9 e 10 bilhões de pessoas em 2050, nos coloca ante um dos maiores desafios globais do século XXI. O relatório do IPBES 2019 mostra que, nos últimos 50 anos, 14 dos 18 serviços ambientais avaliados entraram em declínio e que seus impactos são distribuídos de maneira socialmente desigual entre e dentro de países (IPBES 2019). O aumento na demanda e a pressão sobre os já escassos recursos naturais será inevitável, assim como sérios impactos econômicos.

Salienta-se que essas três crises colocam questões fundamentais sobre nossa sociedade. Uma delas é onde estamos colocando nossos recursos. O exemplo dos Estados Unidos é emblemático. O país gasta anualmente trilhões de dóla- 
res em armas nucleares, máquinas de guerra. Mas um simples vírus de $120 \mathrm{~nm}$ causa a morte de mais de 210 mil norte-americanos em menos de nove meses. Recursos intelectuais, científicos e financeiros estão evidentemente sendo investidos nos lugares errados. A Covid-19 apontou vulnerabilidades importantes em nossa sociedade.

\section{A emergência climática}

O físico sueco Svante Arrhenius em 1896 escreveu um artigo calculando que se dobrássemos a concentração de dióxido de carbono $\left(\mathrm{CO}_{2}\right)$ na atmosfera, a temperatura do planeta subiria $4{ }^{\circ} \mathrm{C}$ por causa do efeito estufa (Arrhenius, 1896). Naquela época, a concentração atmosférica de $\mathrm{CO}_{2}$ era de $280 \mathrm{ppm}$. Atualmente, estamos com concentrações de $\mathrm{CO}_{2}$ em 409 ppm, crescendo a 2.5 ppm por ano. Arrhenius ganhou o prêmio Nobel de Química em 1903. Hoje, com os maiores supercomputadores do mundo, e depois do desenvolvimento da física quântica, se fizermos a mesma conta de Arrhenius, chegaremos a valores muito próximos.

Ao longo dos últimos 150 anos, nossa sociedade fez avanços impressionantes em muitos indicadores (e não tão bons em outros), e a queima de combustíveis fósseis e o desmatamento de florestas fizeram que nossa espécie Homo sapiens fosse responsável pela alteração da composição da atmosfera. Aumentamos a concentração de muitos gases-chave na manutenção do balanço energético de nossa atmosfera, como o $\mathrm{CO}_{2}$, metano $\left(\mathrm{CH}_{4}\right)$, óxido nitroso $\left(\mathrm{N}_{2} \mathrm{O}\right)$, ozônio $\left(\mathrm{O}_{3}\right)$, entre outros. Esses gases absorvem a radiação infravermelha, que é o calor emitido pelo nosso planeta para o espaço. A atmosfera, ao interceptar esses gases, armazena calor adicional e aumenta a temperatura da Terra.

Globalmente, temos várias medidas independentes desse aumento de temperatura, entre eles, o da Nasa GISS, ${ }^{1}$ a série temporal compilada pela NOAA, ${ }^{2}$ e muitas outras. A Figura 1 mostra a evolução da temperatura global de 1850 a 2018 compilada pelo IPCC, com a média terrestre de temperatura e a média de regiões oceânicas ${ }^{3}$ (IPCC SRCCL Shukla et al., 2019). Observamos que o aumento médio de temperatura em áreas continentais já ultrapassa $1,5^{\circ} \mathrm{C}$, enquanto o aumento médio global de temperatura é de $1,1{ }^{\circ} \mathrm{C}$.

O Brasil, com grande área continental, também sofreu forte aumento de temperatura no último século. A Figura 2 mostra o aumento da temperatura média no Brasil de 1890 a 2019, calculado pelo Berkeley Earth Group. ${ }^{4}$ Observamos um aumento médio por década de $24,47^{\circ} \mathrm{C}$ em 1900 para $25,99^{\circ} \mathrm{C}$ em 2017 , portanto um aumento de $1,52^{\circ} \mathrm{C}$ em média no Brasil. Como observamos anteriormente, a média mundial, calculada pelo IPCC é de $1,1^{\circ} \mathrm{C}$ de 1850 a 2010. Alguns estados brasileiros tiveram aumentos significativos na temperatura nos últimos 100 anos, em particular os estados do Nordeste, como por exemplo Piauí $\left(2,27^{\circ} \mathrm{C}\right)$, Maranhão $\left(2,22^{\circ} \mathrm{C}\right)$, Bahia $\left(2,14^{\circ} \mathrm{C}\right)$, Ceará $\left(2,09^{\circ} \mathrm{C}\right)$, Alagoas $\left(2,11^{\circ} \mathrm{C}\right)$. O estado de São Paulo teve aumento de temperatura de $1,92^{\circ} \mathrm{C}$ nesse mesmo período. 


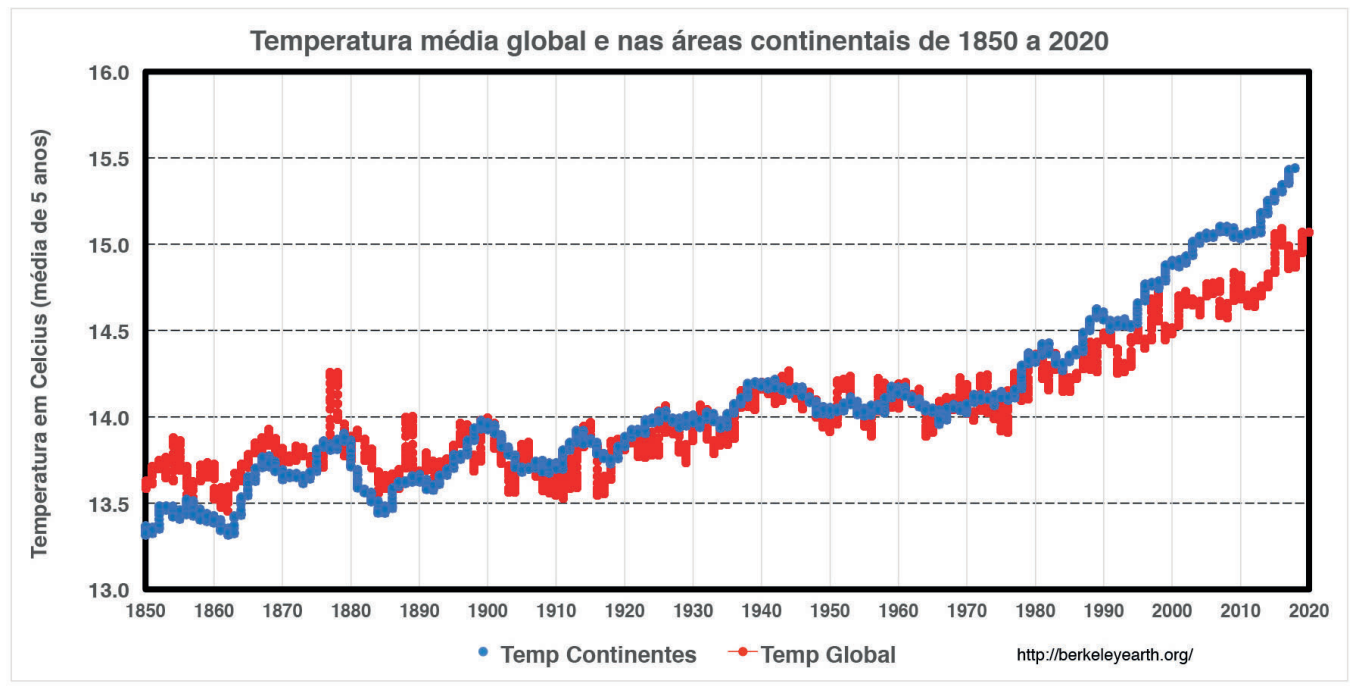

Fonte: Obtida de IPCC SRCCL Shukla et al. (2019).

Figura 1 - Mudança de temperatura global em áreas continentais e na média global, de 1850 a 2018, compiladas pelo IPCC. Disponível em: <https://www. ipcc.ch/srccl/> e <www.berkeleyearth.org $>$.

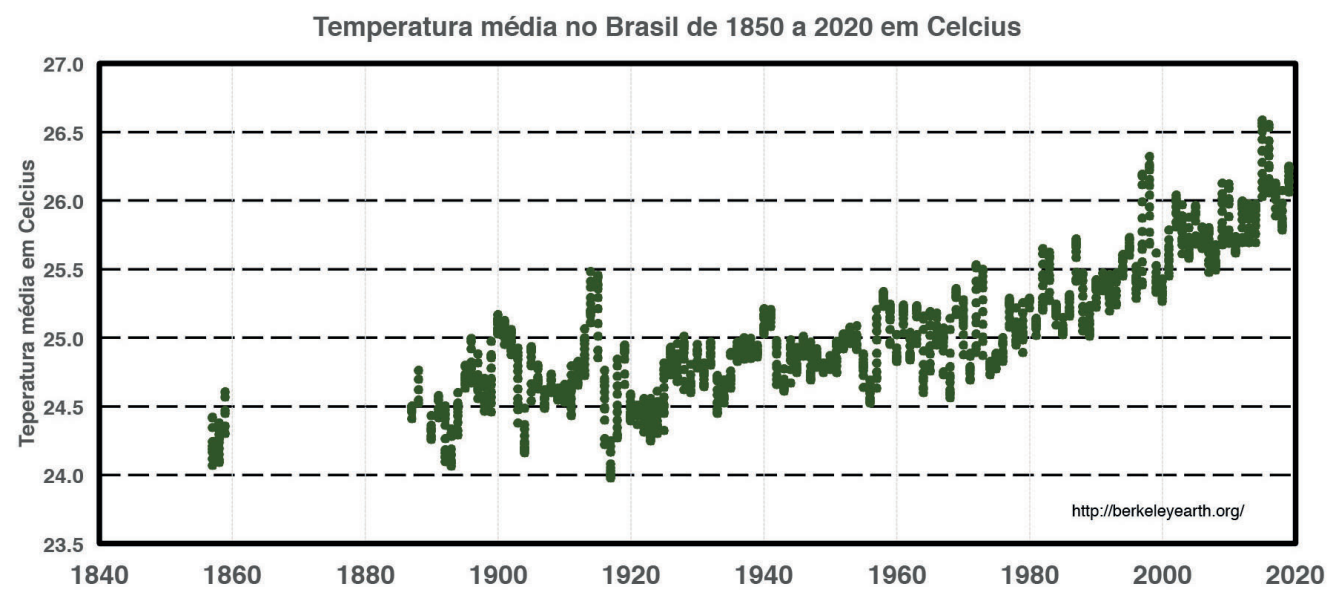

Fonte: Berkeley Earth Group. Disponível em: <http://berkeleyearth.lbl.gov/regions/brazil>.

Figura 2 - Temperatura média no Brasil de 1890 a 2019, em valores anuais e em médias móveis de dez anos.

As mudanças climáticas vão muito além do aumento de temperatura. Alterações na precipitação, circulação atmosférica, eventos climáticos extremos, aumento do nível do mar e outros, também são questões chaves que impactam fortemente nosso sistema socioeconômico. Sendo um país com extensa área costeira, o aumento do nível do mar e da erosão em áreas costeira é particularmente importante.

Para o enfrentamento da emergência climática, o Brasil ratificou o Acordo de Paris, comprometendo-se a reduzir suas emissões de gases de efeito estufa 
em $37 \%$ até 2025 , e $43 \%$ até 2030 , em comparação com emissões verificadas em 2005, e eliminar o desmatamento ilegal da Amazônia até 2025. Também nos comprometemos a aumentar a participação da bioenergia na matriz energética para $18 \%$ até 2030 , restaurar e reflorestar 12 milhões de hectares de florestas, bem como alcançar uma participação de $45 \%$ de energias renováveis na composição da matriz energética em 2030 , além de uma redução em $10 \%$ do consumo de eletricidade. São metas que exigirão esforços consideráveis de toda a sociedade brasileira, inclusive com a eliminação do desmatamento da Amazônia.

O Brasil mostra vulnerabilidades importantes nas áreas ambiental e climática. O observado aumento da frequência e intensidade de eventos climáticos extremos tem impactado sobremaneira nossa população, a economia e o funcionamento dos ecossistemas. Os eventos climáticos extremos impactam a produção agrícola, a infraestrutura costeira, a disponibilidade de recursos hídricos, e a qualidade ambiental das cidades entre muitos outros efeitos. O desmatamento de $11.000 \mathrm{Km}^{2}$ por ano de florestas na Amazônia tem forte impacto no aquecimento global, e afeta o clima regional da América do Sul, com alterações no transporte de vapor de água e do albedo da superfície (Artaxo, 2019).

Importante salientar que a ação humana sobre nosso planeta já transgrediu os limites planetários da integridade da biosfera e dos fluxos biogeoquímicos de nitrogênio e fósforo e está muito próximo de ultrapassar o limite planetário da estabilidade climática (Steffen et al., 2015). Nos sistemas humanos, o IPCC reconhece que as pessoas pobres são as mais vulneráveis aos impactos das mudanças climáticas (IPCC, 2014). O recente relatório do IPCC sobre aquecimento global de $1,5^{\circ} \mathrm{C}$ (IPCC SR $1.5,2018$ ) ressalta a necessidade de eliminar desmatamento de florestas tropicais e reduzir a emissão da queima de combustíveis fósseis em $5 \%$ ao ano até zerar as emissões em 2050. Será necessário remover $\mathrm{CO}_{2}$ da atmosfera a altas taxas (da ordem de -10 bilhões de toneladas $\mathrm{CO}_{2}$ por ano) de 2050 a 2100 para limitar o aquecimento a $1,5^{\circ} \mathrm{C}$. Essa tarefa vai requerer esforços políticos, econômicos e sociais imensos e um sistema de governança inexistente.

E quanto ao futuro climático de nosso planeta? O cenário "business as usual" indica aumento médio da temperatura global da ordem de $4{ }^{\circ} \mathrm{C}$ (IPCC, 2014). Se todos os compromissos dos países acordados no Acordo de Paris forem cumpridos, o aumento médio da temperatura será da ordem de $3.3^{\circ} \mathrm{C}$. Muitos países já anunciaram que não irão cumprir suas metas. A Figura 3 apresenta uma projeção do aumento de temperatura no Brasil no cenário "Business as usual", calculado com a média dos modelos do IPCC pelo Inpe. As projeções foram feitas para a temperatura média do período de 2170-2090, em relação aos valores em 1850.

Vastas regiões do Brasil, inclusive as áreas do Brasil central (Mato Grosso, Goiás, Rondônia e outros estados), onde se concentra a produção agropecuária, podem ter aumento de temperatura de 5 a $6{ }^{\circ} \mathrm{C}$. Poderá haver uma queda na 
precipitação no Nordeste e na parte leste da Amazônia de 20\% a 40\%. Evidentemente, esse cenário, se confirmado, trará profundas alterações para nosso país, como quedas fortes na produção agrícola e na pecuária, fortes na região do Brasil central. O aumento dos eventos climáticos extremos, tais como ondas de calor e secas prolongadas, tem efeitos adicionais ao aquecimento regional, com seus próprios efeitos.

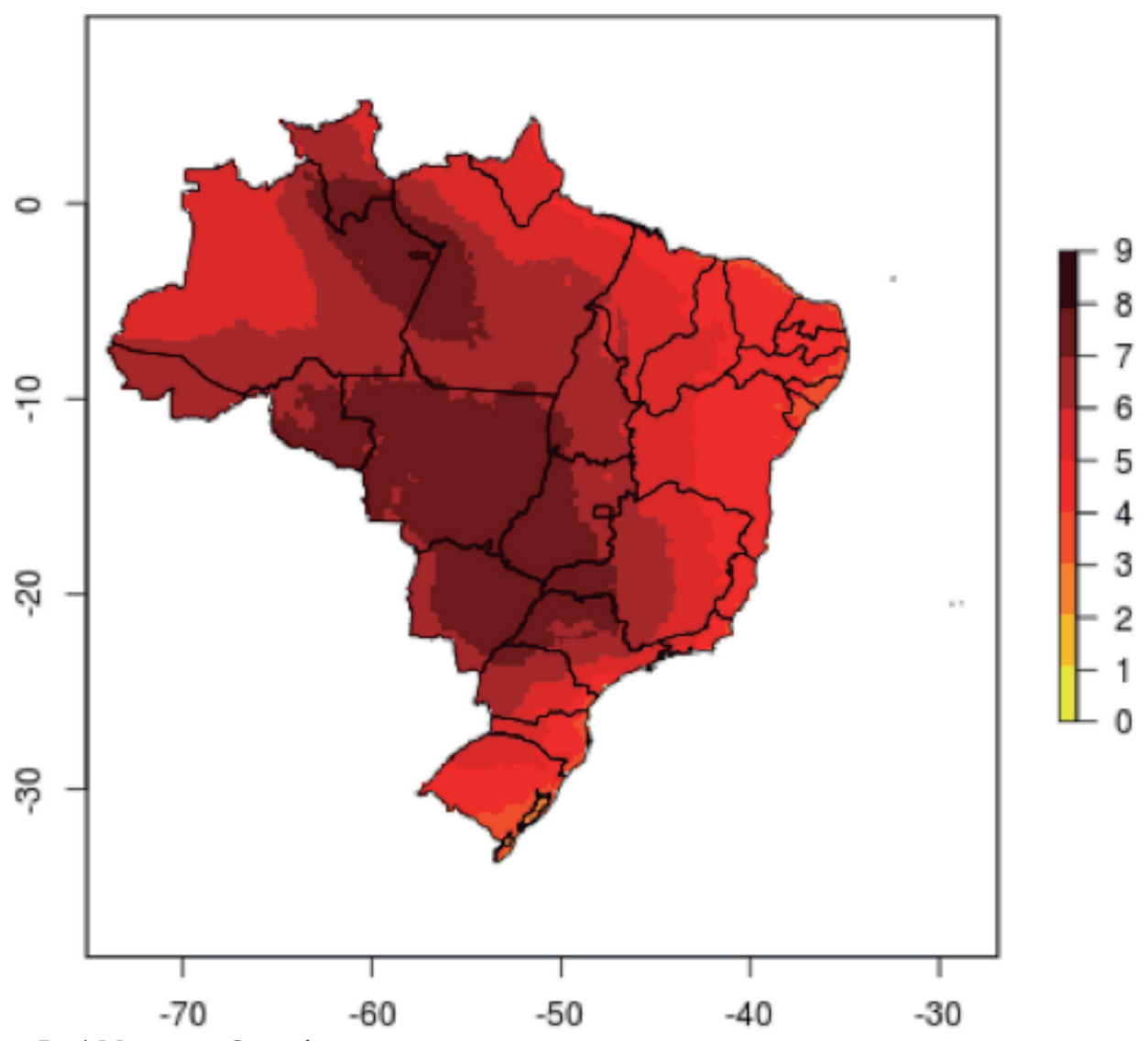

Fonte: José Marengo, Cemadem.

Figura 3 - Projeção do aumento de temperatura no Brasil no cenário "Business as usual", calculado com a média dos modelos do IPCC pelo Cemadem para RCP 8.5. As projeções foram feitas para a temperatura média do período de 2071-2099, em relação aos valores em 1850.

Um ponto que preocupa muito os cientistas é o efeito de aumento de temperatura e redução de precipitação dessa magnitude na floresta Amazônica. A floresta armazena 120-150 bilhões de toneladas de carbono, que correspondem a cerca de doze anos da queima global de combustíveis fósseis. Se uma fração significativa desse carbono for para a atmosfera, o cenário mostrado na Figura 3 poderá ser ainda maior, com impactos muito significativos tanto no clima regional brasileiro quanto no clima global (Nobre et al., 2016). Trabalhos recentes mostram que a Amazônia pode não estar longe desse Tipping point, 
pois a floresta que estava absorvendo grandes quantidades de carbono há dez anos hoje é neutra em termos de emissões, podendo se tornar uma fonte global de $\mathrm{CO}_{2}$ em alguns anos. Isso ocorre pelo aumento da mortalidade das árvores, associada ao incremento de secas nos últimos anos, como as secas de 2005, 2010 e 2015. O clima está se tornando mais extremo em grande parte da Amazônia (Artaxo et al., 2016).

\section{A questão da crise na saúde e mudanças climáticas}

Embora a pandemia de coronavírus esteja provocando grande urgência por causa do perigo que representa, as mudanças climáticas devem ser vistas com igual, e provavelmente ainda maior, urgência, em razão do seu aumento, em todo o planeta e em de muitas maneiras, impactos problemáticos previsíveis e permanentes, e que trarão prejuízos socioeconômicos enormes para nossa sociedade (Heyd et al., 2020). É fundamental que o enfrentamento da pandemia e das mudanças climáticas seja feito de modo conjunto, porque aponta para necessidades de transformações profundas em nossa sociedade, e contém desafios importantes para o Antropoceno. A saúde da população é dependente de aspectos sociais, econômicos, ambientais e de políticas públicas que integrem essa questão como estratégia para o desenvolvimento do país, estado ou município. O Brasil apresenta uma complexa heterogeneidade nas suas regiões, com diversificada distribuição espacial e temporal de determinadas doenças e grande diversidade social, cultural, ecológica e climática que interferem diretamente na resiliência individual e coletiva das populações expostas às mudanças climáticas. Os impactos na saúde resultantes das alterações climáticas globais dependerão do estado geral de saúde das populações expostas que, por sua vez, dependem de condições dos determinantes sociais da saúde como a cobertura de saúde universal, a governança socioambiental, políticas públicas e os rumos do modelo de desenvolvimento do país. O clima tropical e as alterações ecossistêmicas favorecem o desenvolvimento de patógenos.

O Brasil apresenta grande diversidade de animais silvestres que, por sua vez, albergam múltiplos e diferentes microrganismos, muitos desses considerados agentes etiológicos de doenças, tanto para os animais quanto para o homem. Como parte do ciclo de transmissão de inúmeros parasitos, a saúde humana está ligada à saúde dos animais silvestres. As alterações ambientais, incluindo as mudanças climáticas e a perda da biodiversidade, são fatores determinantes para a emergência de doenças oriundas de animais silvestres. Em geral, doenças infecciosas crescem em incidência com maiores temperaturas. Os ecossistemas preservados e em equilíbrio têm um papel importante para a dinâmica e controle de doenças zoonóticas e infecções transmitidas por vetores.

Estima-se que mais de $60 \%$ das doenças infecciosas circulem entre animais e humanos (zoonoses), e que grande parte seja causada por patógenos com origem na vida silvestre. As mudanças ambientais globais têm consequências diretas para o avanço dos patógenos que geram impactos tanto para a saúde pú- 
blica quanto para a conservação de fauna. Dentre eles, classicamente podem ser apontados os agentes etiológicos que causam malária, febre amarela, tuberculose, toxoplasmose, leptospirose, febres hemorrágicas, raiva, brucelose, doença de Chagas, ebola, Sars-CoV-2 e outros coronavírus. As doenças arbovirais, como Dengue, Zika, Febre Chikungunya e Febre Amarela, são importantes ameaças das mudanças globais à saúde pública.

Mudanças de uso do solo e alterações na precipitação e aumento de temperatura estão alterando a migração de mosquitos e facilitando a propagação das chamadas doenças tropicais negligenciadas. O precário sistema de saúde no interior do Brasil faz que o acesso a tratamentos pela população de baixa renda seja difícil, aumentado a mortalidade dessas doenças. A transmissão de doenças associadas aos vetores por mosquitos como Aedes, Culex, Anopheles e outros é influenciada pelo ciclo hidrológico e pela temperatura, que estão mudando em praticamente todo o território nacional.

A queima de biomassa decorrente do desmatamento e de práticas agrícolas faz que os níveis de poluição do ar em áreas remotas tragam significativos efeitos para a saúde pública da região, com a agravamento das doenças respiratórias, principalmente para crianças, idosos e aqueles com enfermidades pregressas, o que aumenta o risco de hospitalização e mortalidade, agravando o cenário pela falta de acesso a serviços de saúde.

O conceito One World, One Health, da Organização Mundial da Saúde (OMS), liga políticas de saúde humana, animal e ambiental. Tem o objetivo de ampliar a visão e as ações para o enfrentamento dos desafios da prevenção de epidemias e epizootias, e manutenção da integridade ecossistêmica em benefício humano e da biodiversidade que os suportam. A pandemia da Covid-19 causada pelo Sars-CoV-2 mostrou a relevância dessa abordagem. A manutenção do equilíbrio climático é chave neste conceito.

A pandemia da Covid-19 nos ensinou que as medidas de antecipação teriam sido consideravelmente mais econômicas do que o nosso presente com ações de enfrentamento, em termos tanto de vidas quanto de recursos economizados. Quando consideramos isso em combinação com o muito mais arriscado, maior e incomensuravelmente mais duradouro, embora mais lento, perturbador impacto das mudanças climáticas, somos chamados a reconhecer as oportunidades presentes para lidar efetivamente com a pandemia e as mudanças climáticas.

\section{A crise da perda de biodiversidade}

Tanto pela ocupação dos hábitats naturais quanto pelas alterações no uso do solo e pela ação humana, estamos observando perda de espécies de modo muito significativo nos ecossistemas terrestres e nos oceanos (International Platform on Biodiversity and Ecosystem Services - IPBS, 2019). O Brasil abriga cerca de 15\% das espécies do planeta em ampla variedade de hábitats. Essa biodiversidade está em seis grandes biomas continentais - Amazônia, Mata Atlântica, Caatinga, Cerrado, Pampa e Pantanal. O país tem ainda seis grandes bacias hidrográfi- 
cas - Amazônica, Tocantins-Araguaia, Parnaíba, São Francisco, Paraíba do Sul e Paraná-Paraguai, e mais de 8 mil quilômetros de costa. O equilíbrio no funcionamento dos ecossistemas depende de alto número de espécies vegetais e animais.

Várias propriedades de sistemas naturais, em particular a biodiversidade, são essenciais para os fluxos de serviços ecossistêmicos que sempre beneficiaram a humanidade, proporcionando segurança hídrica e alimentar, identidade e proteção de valores culturais e garantindo desenvolvimento econômico, social e humano (BPBES, 2018). Em um cenário em direção a um desenvolvimento sustentável, a biodiversidade será decisiva para a mitigação e adaptação das mudanças climáticas, e deverá propiciar novos mecanismos de geração de renda e bem-estar. Por outro lado, em um cenário do atual modelo de desenvolvimento econômico (business as usual), no qual continuamos a nos desenvolver com base na queima de combustíveis fósseis, além de não tomarmos medidas de adaptação à mudança do clima, o declínio dos sistemas naturais de suporte à vida será inevitável, e implicará a aceleração nas mudanças climáticas e impactos negativos sobre nossa sociedade.

\section{Considerações finais}

As mudanças climáticas vêm influenciando alterações no regime de chuvas, na temperatura, no nível e na química de águas costeiras, mudanças na fenologia das plantas, funcionamento de ecossistemas e, além de outros, na distribuição da biodiversidade, inclusive na distribuição de vetores transmissores de doenças. Essas mudanças interagem entre si e com "múltiplos estressores" sociais e ambientais que podem ampliar seus impactos. Porém, muitas dessas dimensões das mudanças climáticas, e suas interações, precisam ser mais bem compreendidas. As fortes alterações no regime de chuvas em todas as regiões do país exigem soluções para minorar os problemas socioeconômicos advindos das secas mais fortes e frequentes, e inundações extremas em grandes áreas.

Como se sabe, o setor agropecuário é fortemente impactado pela alteração no regime de chuvas, e os modelos climáticos com maior qualidade de previsão e cenários baseados em narrativas socioeconômicas calcadas na sustentabilidade podem subsidiar a adaptação desse e de outros importantes setores a um clima alterado. Assim, temos que contribuir para o desenvolvimento de estratégias baseadas na integração das diferentes áreas das ciências, visando atingir a segurança hídrica, alimentar, saúde e energética e a justiça social, buscando estratégias de adaptação climática minimizando os impactos socioeconômicos na sociedade.

Os impactos das mudanças climáticas estão se acelerando rapidamente. Como consequência, é importante reconhecermos a necessidade de fortes ações de mitigação de emissões e de adaptação (WEF, 2020). A ciência tem avançado a passos largos nessa área, mostrando um dinamismo importante em estudos interdisciplinares e transdisciplinares, e voltados à adoção de políticas públicas, seja para as distintas regiões do país, seja para setores específicos como o de água, alimento, transporte e geração e consumo de energia. 
A ciência interdisciplinar, fundamentada na pesquisa de qualidade e continuada, é central para o enfrentamento do maior desafio da humanidade neste século: as mudanças climáticas globais (IPCC, 2014). Entre os possíveis impactos está em evidência a questão de migrações em larga escala no planeta. Algumas regiões habitadas hoje podem não ser capazes de sustentar economicamente sua população no futuro próximo. O Nordeste brasileiro é um exemplo, pois com aumento de temperatura de $5{ }^{\circ} \mathrm{C}$ e queda na precipitação de $30 \%$, a região que é hoje semiárida pode ser tornar árida e requerer grandes migrações internas no Brasil. O mesmo em outras regiões de nosso planeta, trazendo instabilidades políticas, sociais e econômicas em nível global.

Os desafios científicos e tecnológicos para reduzir emissões e manter o aquecimento global máximo em $1,5^{\circ} \mathrm{C}$, como estabelecido no Acordo de Paris, implicam grandes transformações nos sistemas sociais, nas questões territoriais e de uso do solo, energia, infraestrutura e política industrial (IPCC, 2014). O compromisso energético brasileiro em nossa Nationally Determined Contributions, ou Contribuições Nacionalmente Determinadas (NDC) implica desafios de difusão de tecnologias por meio de políticas públicas, instrumentos de mercado e regulação. A transição para uma economia de baixo carbono e maior participação de novas tecnologias energéticas necessitam de reformas profundas no ambiente regulatório e institucional.

O impacto socioeconômico da mitigação e adaptação às mudanças climáticas pode ser muito grande, especialmente em países em desenvolvimento. Mas também pode representar novas oportunidades de negócios e de aumento de eficiência energética, além da possibilidade de construir economias de baixo carbono. É fundamental o papel do setor privado trabalhando junto com a academia e legisladores para minimizar o custo das grandes transformações necessárias em todas as áreas, como energia, edificações, transporte e outros setores. Todos os setores da sociedade precisam dar sua contribuição no desenvolvimento de uma economia de baixo carbono.

É essencial que haja uma integração em todos os níveis, desde o indivíduo, aos municípios, estados, países e globalmente (Artaxo, 2019). Para isso, precisamos de governança integrada às políticas públicas a serem implementadas em todos os níveis. Essas linhas de ação têm que contemplar os objetivos da Agenda 2030 para o desenvolvimento sustentável e são balizadas por estudos globais como os diversos relatórios do Painel Intergovernamental para Mudanças Climáticas (IPCC), as ações propostas pelo IPBES, a Plataforma Intergovernamental sobre Biodiversidade e Serviços Ecossistêmicos, e BPBES, a Plataforma Brasileira de Biodiversidade e Serviços Ecossistêmicos; especialmente focando nas ações transformativas de nossa sociedade.

Em nível global, as Conferência das Nações Unidas sobre Mudanças do Clima (COP) têm um papel importante nos próximos anos e deverão ser substituídas por mecanismos de governança que integrem globalmente as questões de 
saúde, meio ambiente e biodiversidade. Isso deve ser feito com a formulação de políticas públicas baseadas em ciência em todos os níveis (municipal, estadual, nacional e global). O Brasil demonstrou que a redução do desflorestamento da Amazônia pode ser feita rapidamente e com custos modestos, já que o país reduziu o desmatamento anual de $28.000 \mathrm{~km}^{2} \mathrm{em} 2004$ para $4.500 \mathrm{Km}^{2} \mathrm{em} 201 \mathrm{l}$, por meio de políticas públicas consistentes e de longo prazo. A partir de 2012, o desmatamento anual voltou a crescer significativamente e em 2019 atinge o nível de $10.129 \mathrm{~km}^{2}$ no ano. Pressões internacionais pela redução de emissões e pela manutenção das florestas tropicais pode ter efeito importante e o desmatamento na Amazônia voltar a se reduzir.

É possível construir um sistema integrado no qual a biodiversidade seria incorporada como componente central ao processo de desenvolvimento, gerando aumento significativo na capacidade adaptativa dos sistemas naturais e humanos. Nesse cenário, as metas propostas pelos Objetivos de Desenvolvimento Sustentável serão alcançadas, os efeitos negativos do clima estarão presentes, porém o estabelecimento de uma forma de desenvolvimento não predatória à biodiversidade permitirá sua conservação, incluindo um aumento em sua capacidade de resposta às mudanças do clima, com diminuição da contribuição da emissão de carbono pelo desmatamento e o aumento de sua retirada da atmosfera por extensa restauração ecossistêmica, especialmente nas regiões tropicais.

Importante mudarmos radicalmente nosso sistema socioeconômico para um sistema minimamente sustentável, mais justo, com menos desigualdades e atendendo aos Objetivos do Desenvolvimento Sustentáveis (ODS). Um dos caminhos é a construção de sistemas socioecológicos, em que processos e componentes socioeconômicos e biofísicos estejam integrados. O ser humano é considerado parte do sistema natural, ao contrário de ser um agente externo que altere os sistemas ecológicos para seu próprio benefício a curto prazo. Os serviços ecossistêmicos seriam integrados na estrutura do desenvolvimento econômico, com estratégias baseadas em ciência (Artaxo, 2019).

Para lidar com as três crises emergenciais, precisamos desenvolver sistemas de governança que atenda às necessidades de ações integradas em todos os níveis. Na pandemia da Covid-19, observamos que cada país individualmente desenvolveu uma estratégia diferente dos vizinhos, e mesmo estados e municípios no Brasil tiveram estratégias diferentes e às vezes opostas no enfrentamento de uma pandemia que é global. Evidentemente, ações como essas na questão climática não vão funcionar, pois com uma economia global integrada, não há possibilidade de ações fragmentadas, que certamente serão ineficientes.

A Organização das Nações Unidas (ONU) não tem mandato e nem possibilidade de lidar com questões planetárias dessa magnitude. Um novo sistema de governança global terá que ser criado, se quisermos estabilizar a questão climática e estancar a perda de biodiversidade. Teremos que mudar nosso sistema econômico, pois crescimento econômico contínuo em um planeta com recursos 
naturais finitos não é possível. As desigualdades sociais que temos hoje são forte fonte de instabilidade política, social e ambiental. Como resultado da Covid-19, vários países europeus estão discutindo estratégias para o decrescimento econômico com mínimos danos aos seus países. A sustentabilidade em nosso planeta é possível e necessária. Teremos muito trabalho em construí-la nos próximos anos e décadas, pois não temos alternativas.

Agradecimentos - Agradecemos o suporte da Fundação de Amparo à Pesquisa do Estado de São Paulo (Fapesp), projeto temático número 2017/17047-0.

\author{
Notas \\ 1 Disponível em: <https://data.giss.nasa.gov/gistemp/>. \\ 2 Disponível em: <https://www.climate.gov/>. \\ 3 Disponível em: <https://www.ipcc.ch/srccl/>. \\ 4 Disponível em: <http://berkeleyearth.lbl.gov/regions/Brazil>.
}

\title{
Referências
}

AGENDA 2030, ONU Brasil - Agenda 2030 para o Desenvolvimento Sustentável. Disponível em: <http://www.agenda2030.org.br/sobre/>.

ARRHENIUS, S. 1896. XXXI. On the influence of carbonic acid in the air upon the temperature of the ground. The London Edinburgh and Dublin Philosophical Magazine and Journal of Science. doi:10.1080/14786449608620846.

ARTAXO, P. et al. (Ed.) Interactions between Biosphere, Atmosphere and Human Land Use in the Amazon Basin. Berlin: Springer Verlag, Ecological Studies: Analysis and Synthesis 227, 2016. p.478, Hard Cover ISBN 978-3-662-49902-3, 227. DOI: 10.1007/978-3-662-49902-3. 2016.

ARTAXO, P. Working together for Amazonia. Editorial Science Magazine, v.363, Issue 6425, doi: 10.1126/science.aaw6986, 2019.

BPBES. $1^{\circ}$ Diagnóstico Brasileiro de Biodiversidade e Serviços Ecossistêmicos. Sumário para tomadores de decisão. 2018. Disponível em: <https://www.bpbes.net.br/wp-content/uploads/2018/11/Sum\%C3\%Alrio-para-Tomadores-de-Decis\%C3\%A3o-BPBES-1.pdf>.

HEYD, T. Covid-19 and climate change in the times of the Anthropocene. The Anthropocene Review, p.'l-16, 2020. https://doi.org/10.1177/2053019620961799.

IPBES. Global assessment report of the Intergovernmental Science-Policy Platform on Biodiversity and Ecosystem Services. UN-IPBES, 2019. https://ipbes.net/.

IPCC. Climate Change 2014: Impacts, Adaptation, and Vulnerability. Part A: Global and Sectoral Aspects. Contribution of Working Group II to the Fifth Assessment Report of the Intergovernmental Panel on Climate Change. Cambridge: Cambridge 
University Press, IPCC AR5 Synthesis Report, 2014. Disponível em: <https://www. ipcc.ch/report/ar5/syr/>.

IPCC SR 1.5. Global Warming of $1.5^{\circ} \mathrm{C}$ : an IPCC special report on the impacts of global warming of $1.5^{\circ} \mathrm{C}$ above pre-industrial levels and related global greenhouse gas emission pathways, in the context of strengthening the global response to the threat of climate change, sustainable development, and efforts to eradicate poverty. Summary for Policymakers. 2018. Disponível em: <https://www.ipcc.ch/sr15/>.

NOBRE, C. A. et al. Land-use and climate change risks in the Amazon and the need of a novel sustainable development paradigm. Proceedings of the National Academy of Sciences, v.113, p.10759-68, 2016.

STEFFEN, W. et al. Planetary boundaries: guiding human development on a changing planet. Science, n.347, 2015. DOI 10.1126/science.1259855.

WEF - World Economic Forum Global Risks Report 2020. Disponível em: <https:// www.weforum.org/global-risks/reports>.

RESUMO - Estamos entrando em uma era em que nosso planeta e nossa sociedade estão enfrentando crises importantes. Convivemos simultaneamente com três emergências importantes: 1) a crise na saúde, intensificada com a pandemia da Covid-19; 2) a crise de perda de biodiversidade; e 3) a emergência climática. Salienta-se que essas crises têm ligações profundas entre si, e diferenças também importantes, mas todas provocam impactos sociais e econômicos fortes e afetam o planeta globalmente. Elas são resultado de um modelo econômico que privilegia o desenvolvimento a qualquer custo, o lucro muito rápido, mesmo à custa da sustentabilidade. A crise da Covid-19 colocou questões importantes do ponto de vista de falta de governança global. Por sua vez, a crise climática tem potencial para danos socioeconômicos muito fortes, e seus efeitos já são facilmente visíveis. A perda de biodiversidade coloca em risco nossa segurança alimentar, bem como o equilíbrio do sistema terrestre. A Amazônia, por exemplo, contém milhares de vírus em sua fauna e flora, e a continuar o processo desenfreado de sua ocupação, novos vírus similares ao Sars-CoV-2 possivelmente entrarão em contato com nossa sociedade. Sair dessas três crises requer mudanças drásticas em nosso sistema econômico, insustentável em seu formato atual. Crescimento econômico contínuo em um planeta com recursos naturais finitos não é possível. A desigualdade em países em desenvolvimento e mesmo estre nações é explosiva e injusta. Precisaremos de um novo sistema de governança global que seja capaz de harmonizar medidas dos diferentes países, estados e municípios. A sustentabilidade em nosso planeta é possível e necessária. Teremos muito trabalho em construí-la nos próximos anos, comunidade científica, sociedade, governos e outros agentes. Não temos alternativas senão construir uma nova sociedade, mais justa e sustentável.

PALAVRAS-CHAVE: Covid-19, Biodiversidade, Mudanças climáticas, Amazônia.

ABSTRACT - We are entering an era in which our planet and our society face major crises. We live simultaneously with three important emergencies: 1) the health crisis, intensified by the Covid-19 pandemic; 2 ) the biodiversity loss crisis; and 3 ) the climatic emergency. It should be noted that these crises have profound links between them, and also 
important differences, but they all have strong social and economic impacts and affect the planet globally. They are the result of an economic model that favors development at any cost and very quick profits even at the expense of sustainability. The Covid-19 crisis posed important questions from the viewpoint of a lack of global governance. In turn, the climate crisis has the potential for very serious socio-economic damage, and its effects are already clearly visible. The loss of biodiversity puts our food security at risk, and the balance of the terrestrial system as well. The Amazon, for example, harbors thousands of viruses in its fauna and flora, and if the unrestrained process of occupation continues, new viruses similar to Sars-CoV-2 will possibly come into contact with our society. Overcoming these three crises requires drastic changes in our economic system, unsustainable in its current format. Continued economic growth on a planet with finite natural resources is not possible. Inequality in developing countries and even between nations is explosive and unfair. We will need a new system of global governance that is able to harmonize measures from different countries, states and municipalities. Sustainability on our planet is possible and necessary. We - the scientific community, society, governments and other stakeholders -will have a lot of work ahead of us in the coming years. We have no alternative but to build a new, more just and sustainable society.

KErWords: Covid-19, Biodiversity, Climate change, Amazonia.

Paulo Artaxo é professor do Instituto de Física da Universidade de São Paulo.

@ - artaxo@if.usp.br / https://orcid.org/0000-0001-7754-3036.

Recebido em 5.10.2020 e aceito em 13.10.2020.

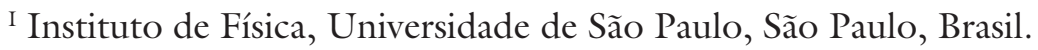

